\title{
Neuromuscular Electrical Stimulation and an Ottoman-Type Seat Effectively Improve Popliteal Venous Flow in a Sitting Position
}

\author{
Hironobu MORITA1', Chikara ABE11, Kunihiko TANAKA1, Mitsuo SHIRATORI2, \\ Masato OGURI2, and Toshikazu SHIGA ${ }^{3}$ \\ 1Department of Physiology, Gifu University Graduate School of Medicine, 1-1 Yanagido, Gifu, 501-1194 Japan; 2Toyota Development \\ Center, Toyota Motor Corporation, 1 Toyota-cho, Toyota, 471-8572 Japan; and 3Omron Healthcare Corporation, 24 Yamanouchi \\ Yamanoshita-cho, Kyoto, 615-0084 Japan
}

\begin{abstract}
The aim of this study was to examine the effects of the sitting posture on the lower limb venous flow and to explore the beneficial effects of neuromuscular electrical stimulation (NMES) and an ottoman-type seat on the venous flow. Healthy adult volunteers without a history of lower limb surgery or thromboembolism were recruited, and the flow velocity, cross-sectional area, and flow volume of the popliteal vein were measured using Doppler ultrasound. A posture change from the prone position to the sitting position on the ottoman-type seat decreased the flow velocity and increased the cross-sectional area of the popliteal vein, and the flow volume was not altered over
\end{abstract}

$120 \mathrm{~min}$. The flow velocity was further decreased, and the crosssectional area was further increased by subjects sitting on a regular driving seat when compared with the values obtained with an ottoman-type seat. The popliteal flow velocity in the NMES leg was significantly higher than in the non-NMES leg throughout the 120-min testing in the sitting position, but no difference in the cross-sectional area was found between the NMES and the non-NMES legs. Thus lower limb venous stasis elicited by the sitting posture was improved by the ottoman-type seat and NMES.

Key words: muscle contraction, electrical stimulation, blood flow.

Scurr et al. [1] reported that after long-haul air travel, symptomless deep venous thrombosis (DVT) develops in $10 \%$ of passengers, which is a surprisingly large proportion. The sitting posture in an airplane exposes passengers to the combined effects of movement restriction and gravitational pressure gradient between the heart and the lower limbs; this might enhance venous pooling/stasis, thereby increasing the chances of the development of DVT. Accordingly, the effects of sitting posture on lower limb hemodynamics have been a matter of great interest, and it was found that the venous flow velocity in the lower limbs was decreased and the diameter of the popliteal vein was increased by a sitting posture [2-5]. Devices that increase the venous flow velocity are thought to be effective in reducing venous stasis, and many techniques, including neuromuscular electrical stimulation (NMES) [6-8], have been proposed for increasing venous flow velocity. The metabolic demands of exercising skeletal muscles are met by increasing their blood flow, thereby supplying additional oxygen; this is a well-known phenomenon. This is also true in the case of passive electrical stimulation [9, 10]. Furthermore, venous flow velocity increases in response to muscle contraction, and the increased velocity contributes to venous expulsion [10]. NMES increases the venous flow velocity in a stimulation-dependent manner, and the effect lasts for $4 \mathrm{~h}$ [7]. However, the extent to which NMES can improve the suppression of venous flow induced by a sitting posture remains unclear. To examine this, the popliteal venous flow velocity was measured consecutively in the horizontal prone position and then in the sitting position in both NMES and non-NMES legs. Furthermore, the effects of an ottoman-type seat on lower limb hemodynamics were also examined.

\section{Methods}

This study was approved by the ethics committee of our institution. Twenty-one healthy adult volunteers (14 men and 7 women aged 20-50) were recruited; the subjects had no history of lower limb surgery or thromboembolism.

In 15 subjects ( 8 men and 7 women aged $20-50$ ), one lower limb was randomly selected for stimulation, and a pair of stimulating pads $(80 \times 60 \mathrm{~mm})$ was attached to the surface of the gastrocnemius muscle; the other limb served as a control. The subject lay down in a horizontal prone position, and a 30-min resting period was allowed

Received on Mar 15, 2006; accepted on Apr 18, 2006; released online on Apr 20, 2006; doi:10.2170/physiolsci.SC002006

Correspondence should be addressed to: Hironobu Morita, Department of Physiology, Gifu University Graduate School of Medicine, 11 Yanagido, Gifu, 501-1194 Japan. Phone: +81-58-230-6300, Fax: +81-58-230-6302, E-mail: morita@cc.gifu-u.ac.jp 


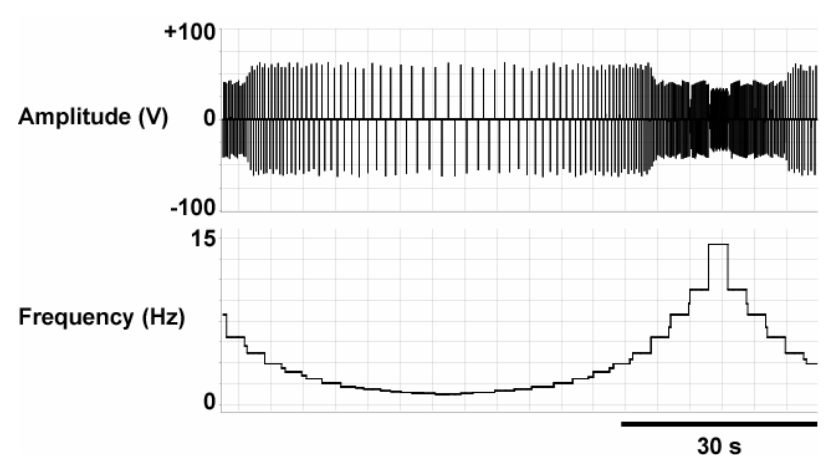

Fig. 1. Original record of the amplitude and frequency of NMES.

prior to the beginning of the experimental protocol. After a period of acclimatization, the baseline venous flow in the popliteal vein was assessed. A duplex scanning of the popliteal region was performed using the Toshiba Nemio SSA-550A ultrasound machine fitted with a $7.5 \mathrm{MHz}$ linear scan head (Toshiba Medical, Tokyo). The angle of insonation (the angle between the direction of the Doppler wave and the vessel) was maintained at approximately $60^{\circ}$, and the sample volume length was maintained at $25 \%-50 \%$ of the vessel diameter. The settings (gain and contrast) were optimized after initial evaluations and then maintained constant. The Doppler waveform of the flow velocity in the vein was obtained by simultaneous realtime B-mode and gated Doppler ultrasonography. It was stored in the memory and analyzed by software that allows automated enveloping of the profile of the velocity waveform (outline) with an estimation of its peak $\left(V_{\max }\right)$ and time-averaged maximum flow velocities over $8 \mathrm{~s}$ $\left(T A V_{\max }\right)$. These 2 variables, the cross-sectional area of the vein and the flow volume (the product of $T A V_{\max }$ and cross-sectional area) were studied. At least 3 measurements were performed for each time point and then averaged.
After the data for the prone position was acquired, the subject sat on a driving seat whose angle of backrest and height, angle, and length of flange could be optimized by the subject. In this posture, the heels were positioned 40$45 \mathrm{~cm}$ below the hip joint. After the optimization of the seat, NMES was started (repeated in a $10 \mathrm{~min}$ on and 5 min off sequence). The NMES parameters were as follows: duration, $0.2 \mathrm{~ms}$; amplitude, $\sim \pm 60 \mathrm{~V}$; a biphasic waveform; and frequency, $1-15 \mathrm{~Hz}$ (Fig. 1). The stimulation amplitude was gradually increased to an intensity that was just sufficient to create a slightly visible muscle twitch. The subject maintained the sitting posture for 120 $\mathrm{min}$, and the popliteal venous flow data were obtained every $30 \mathrm{~min}$. The flow data of the NMES leg was obtained during the "stimulation on" period.

The popliteal venous hemodynamic values of the other group of 9 subjects ( 9 men aged 24-50 years, including 3 from the first study) were also measured while they were seated on ottoman-type seats for $120 \mathrm{~min}$. On the ottoman-type seat, the heels were positioned $0-10 \mathrm{~cm}$ below the hip joint.

All values are presented as means \pm SEM. The data in Fig. 2 were analyzed by two-way repeated-measure ANOVA; the groups of treatments (NMES leg, non-NMES leg, and ottoman-type seat) and the time course (prone position and 30, 60, 90, and $120 \mathrm{~min}$ in the sitting position) were the factors. If the $\mathrm{F}$ ratio indicated a statistical significance, the Tukey-Kramer procedure was applied to compare the among- and within-group means. The significance level of the Tukey-Kramer comparison was set at $P$ $<0.05$.

\section{Results}

A posture change from the prone to the sitting position on the ottoman-type seat decreased the popliteal venous flow velocity. $T A V_{\max }$ significantly decreased to $12.5 \pm 1.8$ $\mathrm{cm} / \mathrm{s}$, from $22.2 \pm 3.2 \mathrm{~cm} / \mathrm{s}$; this reduction of velocity was
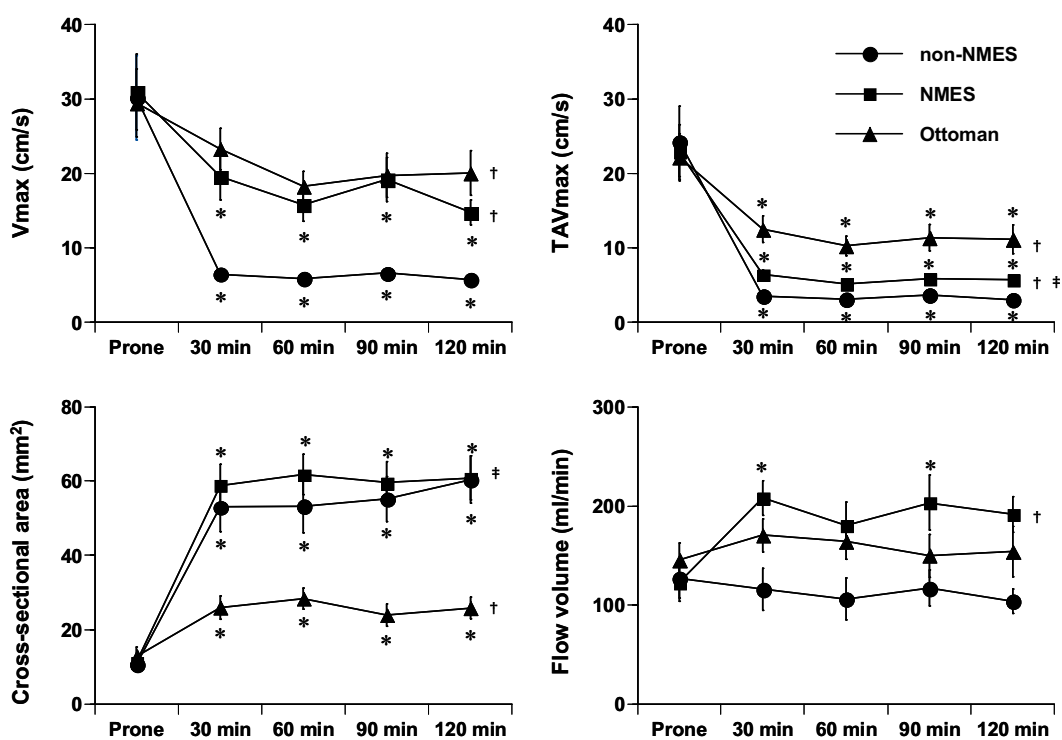

Fig. 2. Summarized data of the peak velocity $\left(V_{\max }\right)$, mean velocity $\left(T A V_{\max }\right)$, cross-sectional area (Area), and flow volume (Flow) of the popliteal vein. Data are presented as mean \pm SEM. Circles: variables measured in non-NMES legs; squares: variables measured in NMES legs; triangles: variables measured in legs on the ottoman-type seat. $* P<0.05$ : variables are significantly different from those in the prone position. $\dagger P<0.05$ : variables measured in NMES legs or those on the ottoman-type seat are significantly different from the variables measured in non-NMES legs. $\ddagger P<0.05$ : variables measured in legs on the ottoman-type seat are significantly different from those in NMES legs. 
not recovered throughout the 120-min experimental period (Fig. 2). In contrast to the velocity, the cross-sectional area of the popliteal vein significantly increased to $26.0 \pm$ $3.2 \mathrm{~mm}^{2}$, from $12.9 \pm 2.4 \mathrm{~mm}^{2}$, at $30 \mathrm{~min}$, and this level was maintained for $120 \mathrm{~min}$. Because of the combined effect of the decreased $T A V_{\max }$ value and the increased cross-sectional area, the flow volume of the popliteal vein was not affected by sitting on an ottoman-type seat.

The hemodynamic changes in the popliteal vein were more serious while subjects were sitting on a regular driving seat when compared with those observed on an ottoman-type seat. In the non-NMES leg, $V_{\max }$ and $T A V_{\max }$ decreased to $6.5 \pm 0.5 \mathrm{~cm} / \mathrm{s}$, from $30.2 \pm 5.7 \mathrm{~cm} / \mathrm{s}$, and to 3.5 $\pm 0.4 \mathrm{~cm} / \mathrm{s}$, from $24.3 \pm 4.8 \mathrm{~cm} / \mathrm{s}$, respectively. The crosssectional area of the popliteal vein significantly increased to $53.1 \pm 6.7 \mathrm{~mm}^{2}$, from $10.8 \pm 1.0 \mathrm{~mm}^{2}$. Because of the combined effect of the decreased $T A V_{\max }$ value and the increased cross-sectional area, the flow volume of the popliteal vein was not affected by the sitting posture (changed to $115.9 \pm 21.1 \mathrm{ml} / \mathrm{min}$, from $126.9 \pm 19.9 \mathrm{ml} /$ $\min )$.

$V_{\max }$ and $T A V_{\max }$ of the NMES leg were significantly higher than those of the non-NMES leg throughout the 120 -min sitting period. There was no difference in the cross-sectional area between the NMES leg and the nonNMES leg, but the flow volume was significantly greater in the NMES leg than in the non-NMES leg. Furthermore, the combination of NMES and the sitting posture significantly increased the popliteal venous flow volume when compared with that in the prone position. Although NMES significantly improved the popliteal venous flow velocity, the flow velocity in the prone position was not fully recovered by NMES; i.e., the 120 -min averages of $V_{\max }$ and $T A V_{\max }$ were $68 \pm 10 \%$ and $32 \pm 4 \%$ of those in the prone position, respectively.

\section{Discussion}

The major findings of the present study are as follows: (i) the sitting posture significantly decreased the popliteal venous flow velocity, but the cross-sectional area was increased, and the flow volume was not affected; (ii) NMES significantly improved the venous flow velocity and increased the flow volume, but the flow velocity did not fully recover to the level present in the prone position; and (iii) lifting up the lower thigh using the ottoman-type seat also improved the venous flow, along with an increase in the flow velocity and a decrease in the cross-sectional area.

The sitting posture is known to reduce the lower limb venous flow velocity $[2,3]$, probably a result of the gravitational pressure gradient between the heart and lower limbs, vein flexion causing obstruction, and inactivation of the muscle pump. Thus the main objective of the mechanical prophylaxis for DVT is an enhancement of the deep venous flow velocity for decreasing stasis. For this purpose, intermittent pneumatic compression and graduated compression stockings are commonly used [11-14]. The former significantly increases the venous flow velocity [11-13]; however, it is neither convenient nor expensive. In contrast, the latter has no significant effect on the venous flow velocity $[13,14]$, though it significantly decreases the incidence of DVT associated with long-haul air travel $[1,15]$.

An activation of the muscle pump by NMES is another candidate technique for mechanical prophylaxis [6-8]. Lyons et al. [8] demonstrated that NMES of the calf muscle increases the popliteal venous flow velocity in a stimulation-dependent (duration and frequency) manner. Kaplan et al. [7] showed the effects of NMES over an extended period; in their study, the femoral and popliteal venous flow velocities in the NMES leg were significantly higher than those in the non-NMES leg throughout the $4 \mathrm{~h}$ of testing. However, the extent to which NMES can improve the decreased velocity has not been clarified. In the present study, we measured the popliteal venous flow for $120 \mathrm{~min}$ in a sitting position and clearly demonstrated the beneficial effects of NMES. In the non-NMES legs, $V_{\max }$ and $T A V_{\max }$ were decreased to $27 \pm 3 \%$ and $20 \pm 3 \%$, respectively, by the sitting posture. On the other hand, in the NMES legs, $V_{\max }$ and $T A V_{\max }$ recovered to $68 \pm 10 \%$ and $32 \pm 4 \%$ of those in the prone position, respectively. The effect of NMES on popliteal venous flow velocity was greater in the $V_{\max }$ compared with the $T A V_{\max }$. The $T A V_{\max }$ is inversely proportional to the cross-sectional area, since the $T A V_{\max }$ is the quotient of the flow volume divided by the cross-sectional area. The increased gravitational pressure gradient on the compliant walls of the lower limb vein would result in the enlargement of the cross-sectional area, which was not improved by NMES. Thus the improvement of $T A V_{\max }$ was small. On the other hand, an NMES-induced muscle contraction instantaneously expels venous blood, and this would directly improve $V_{\max }$. The increased $V_{\max }$ would contribute to wash away activated clotting factors and then prevent thrombus formation.

During NMES, no subject complained of pain or discomfort. Stimulation parameters and electrode size are the important factors that determine the perceived comfort during NMES. A symmetrical biphasic waveform, short pulse duration, graded onset of stimulation, and large electrodes are required for comfort and less fatigue [16], and these stimulation parameters were employed in the present study.

The ottoman-type seat also improved the popliteal venous flow, i.e., $V_{\max }$ and $T A V_{\max }$ were significantly higher and the cross-sectional area was smaller than those observed with the regular seat. The difference may arise mainly as a result of the smaller gravitational pressure gradient because with the ottoman-type seat, the heel was positioned approximately $40 \mathrm{~cm}$ higher than with the reg- 
ular seat. Ashby et al. [2] measured the common femoral venous flow velocity and diameter and found that over the range of $6^{\circ}$ leg down to $12^{\circ}$ leg up, there is a linear increase in the mean peak velocity and a decrease in the mean diameter. If the compliance and length of the vein are constant, the cross-sectional area is altered in proportion to the venous pressure. In the sitting position, the lower limb venous pressure should be increased by an increased gravitational pressure gradient, leading to an increase in the cross-sectional area. If the flow volume is constant, the increased cross-sectional area leads to a decrease in the flow velocity because the flow volume is a product of the cross-sectional area and flow velocity. This may be the case with the change in the popliteal venous flow observed in the sitting position. Thus with a decrease in the gravitational pressure gradient when an ottomantype seat is used, the cross-sectional area was decreased and the flow velocity was increased when compared with those observed when a regular seat was used. The product of these two variables (flow volume) was not altered.

In conclusion, NMES could improve the lower limb venous flow in the sitting position, though the flow velocity in the prone position was not fully recovered by it.

This study was supported by the "Ground-based Research Program for Space Utilization" promoted by the Japan Space Forum; a Grant-in-Aid for Scientific Research from the Ministry of Education, Science, Sports and Culture of Japan; a project grant from Gifu University; and grants from the Toyota Motor Corporation and Omron Health Care Corporation.

\section{REFERENCES}

1. Scurr JH, Machin SJ, Bailey-King S, Mackie IJ, McDonald S, Smith PD. Frequency and prevention of symptomless deep-vein thrombosis in long-haul flights: a randomised trial. Lancet. 2001;357:1485-9.

2. Ashby EC, Ashford NS, Campbell MJ. Posture, blood velocity in common femoral vein, and prophylaxis of venous thromboembolism. Lancet.
1995;345:419-21.

3. Delis KT, Knaggs AL, Sonecha TN, Zervas V, Jenkins MP, Wolfe JH. Lower limb venous haemodynamic impairment on dependency: quantification and implications for the "economy class" position. Thromb Haemost. 2004;91: 94150.

4. Essandoh LK, Duprez DA, Shepherd JT. Postural cardiovascular reflexes: comparison of responses of forearm and calf resistance vessels. J Appl Physiol. 1987;63:1801-5.

5. Shvartz E, Gaume JG, White RT, Reibold RC. Hemodynamic responses during prolonged sitting. J Appl Physiol. 1983;54:1673-80.

6. Faghri PD, Van Meerdervort HF, Glaser RM, Figoni SF. Electrical stimulationinduced contraction to reduce blood stasis during arthroplasty. IEEE Trans Rehabil Eng. 1997;5:62-9.

7. Kaplan RE, Czyrny JJ, Fung TS, Unsworth JD, Hirsh J. Electrical foot stimulation and implications for the prevention of venous thromboembolic disease. Thromb Haemost. 2002;88:200-4.

8. Lyons GM, Leane GE, Grace PA. The effect of electrical stimulation of the calf muscle and compression stocking on venous blood flow velocity. Eur J Vasc Endovasc Surg. 2002;23:564-6.

9. Daniels JW, Stebbins CL, Longhurst JC. Hemodynamic responses to static and dynamic muscle contractions at equivalent workloads. Am J Physiol Regul Integr Comp Physiol. 2000;279:R1849-55.

10. Valic Z, Buckwalter JB, Clifford PS. Muscle blood flow response to contraction: influence of venous pressure. J Appl Physiol. 2005;98:72-6.

11. Delis KT, Slimani G, Hafez HM, Nicolaides AN. Enhancing venous outflow in the lower limb with intermittent pneumatic compression. A comparative haemodynamic analysis on the effect of foot vs. calf vs. foot and calf compression. Eur J Vasc Endovasc Surg. 2000;19:250-60.

12. Kakkos SK, Szendro G, Griffin M, Daskalopoulou SS, Nicolaides AN. The efficacy of the new SCD response compression system in the prevention of venous stasis. J Vasc Surg. 2000;32:932-40.

13. Keith SL, McLaughlin DJ, Anderson FA, Jr., Cardullo PA, Jones CE, Rohrer MJ, Cutler BS. Do graduated compression stockings and pneumatic boots have an additive effect on the peak velocity of venous blood flow? Arch Surg. 1992;127:727-30.

14. Macklon NS Greer IA. Technical note: compression stockings and posture: a comparative study of their effects on the proximal deep veins of the leg at rest. $\mathrm{Br}$ J Radiol. 1995;68: 515-8.

15. Belcaro G, Geroulakos G, Nicolaides AN, Myers KA, Winford M. Venous thromboembolism from air travel: the LONFLIT study. Angiology. 2001;52:36974.

16. Lyons GM, Leane GE, Clarke-Moloney M, O'Brien JV, Grace PA. An investigation of the effect of electrode size and electrode location on comfort during stimulation of the gastrocnemius muscle. Med Eng Phys. 2004;26:873-8. 